\title{
Phase recovering algorithms for extended objects encoded in digitally recorded holograms
}

\author{
P. Picart ${ }^{1, \mathrm{a}}$, P. Tankam² ${ }^{2}$ Z. Peng ${ }^{3}$, and J.C. $\mathrm{Li}^{3}$ \\ ${ }^{1}$ LAUM CNRS, Université du Maine, Avenue Olivier Messiaen, 72085 LE MANS Cedex 9, France \\ ${ }^{2}$ École Nationale Supérieure d'Ingénieurs du Mans, rue Aristote, 72085 Le Mans Cedex 9, France \\ ${ }^{3}$ Kunming University of Science and Technology, Kunming, 650093, P.R. China
}

\begin{abstract}
The paper presents algorithms to recover the optical phase of digitally encoded holograms. Algorithms are based on the use of a numerical spherical reconstructing wave. Proof of the validity of the concept is performed through an experimental off axis digital holographic set-up. Two-color digital holographic reconstruction is also investigated. Application of the color set-up and algorithms concerns the simultaneous two-dimensional deformation measurement of an object submitted to a mechanical loading.
\end{abstract}

\section{Introduction}

In digital holography, the numerical reconstruction of the object encoded using optical interferences is usually based on the discrete version of the Fresnel transform, on the computation of the convolution formula of diffraction [1-3] or on the angular spectrum method [4]. The discrete Fresnel transform is very well adapted to large objects, i.e. objects with lateral dimensions quite greater than that of the recording area. With this method, the sampling pitch in the reconstructed plane depends on the wavelength of the light used for the recording, on the diffraction distance and on the number of data points used for the computation. However, such algorithm is not suitable for digital color holography except if one uses a zero padding adapted to the wavelength [5]. With convolution, the field of view conserves the same physical dimension as that of the recording. Thus this approach is limited to very small objects and a convolution algorithm designed for large object has not been proposed in the literature.

This paper proposes two new alternative algorithms based on the convolution approach, and allowing the reconstruction of a large object size variety. We present application of the method in the field of mechanical deformation measurements.

\section{Algorithms}

The algorithms are based on the use of a virtual spherical wave as a reconstructing wave whereas classically a plane wave is considered. So, the reconstruction distance $d_{R}$ that must be put into the algorithm is modified and depends on the physical distance between object and sensor and on the

\footnotetext{
a e-mail : pascal.picart@univ-lemans.fr
} 
curvature radius of the virtual wave according to $1 / d_{R}=-1 / d_{0}+1 / R_{c}\left(d_{0}\right.$ physical distance, $R_{c}$ curvature radius). The reconstructing wave can be chosen to be a Rayleigh-Sommerfeld spherical wave as explicated in Eq. 1:

$$
w\left(x, y, R_{c}\right)=\frac{i R_{c}}{\lambda} \frac{\exp \left[2 i \pi \operatorname{sgn}\left(R_{c}\right) / \lambda \sqrt{R_{c}^{2}+x^{2}+y^{2}}\right]}{R_{c}^{2}+x^{2}+y^{2}}
$$

Here, $\operatorname{sgn}(\ldots)$ is the sign function, $i=\sqrt{-1}$ and $\lambda$ is the wavelength of the light. The main consequence of this is that the object bandwidth becomes adapted to that of the reconstruction kernel. The choice of the curvature radius is governed by the transversal magnification, $\gamma$, between the reconstructed object and the extended physical object and it is found to be given by $R_{c}=\gamma d_{0} /(\gamma-1)$. Furthermore the transversal magnification between reconstructed object and real one is given by $\gamma=-d_{R} / d_{0}$. Since the convolution approach sets the size of the reconstructed horizon, consider that it is $\left(L p_{x}, K p_{y}\right)$. The transversal magnification $\gamma$ must be chosen according to the ratio $L p_{x} / \Delta A_{x}$ (or $K p_{y} / \Delta A_{y}$ ), where $\left\{\Delta A_{x}, \Delta A_{y}\right\}$ are respectively the object sizes along the $x$ and $y$ directions. The transfer function associated to the spatial spectral filtering can be chosen in two ways: the first is based on the use of a modified version of the angular spectrum transfer function and the last is based on the spatially phase biased Fresnel impulse response of free space propagation. In the first case, we chose a transfer function as a modified version of the angular spectrum transfer function according to Eq. $2\left(u_{0}, v_{0}\right.$ spatial frequencies of the reference wave):

$$
\tilde{H}\left(u, v, d_{R}\right)=\left\{\begin{array}{c}
\exp \left[2 i \pi d_{R} / \lambda \sqrt{1-\lambda^{2}\left(u-u_{0}\right)^{2}-\lambda^{2}\left(v-v_{0}\right)^{2}}\right] \\
\quad \text { if }\left|u-u_{0}\right| \leq|\gamma| \Delta A_{x} / 2 \lambda d_{R} \\
\quad \text { and }\left|v-v_{0}\right| \leq|\gamma| \Delta A_{y} / 2 \lambda d_{R} \\
\text { elsewhere }
\end{array}\right.
$$

In the last case, the transfer function is the Fourier transform of the spatially phase biased Fresnel impulse response of free space propagation which is given according to Eq. 3. These two algorithms allow the reconstruction of any horizon, from that of the sensor to zero-padded horizons such as $2048 \times 2048$ pixels.

$$
h\left(x, y, d_{R}\right)=\left\{\begin{array}{l}
\exp \left[\frac{i \pi}{\lambda d_{R}}\left(x^{2}+y^{2}\right)+2 i \pi\left(u_{0} x+v_{0} y\right)\right] \\
\text { if }|x| \leq|\gamma| \Delta A_{x} / 2 \text { and }|y| \leq|\gamma| \Delta A_{y} / 2 \\
0 \quad \text { elsewhere }
\end{array}\right.
$$

\section{Experimental set-up}

The proposed concept is validated using an experimental off axis digital holographic set-up, based on a Mach-Zehnder architecture (Fig. 1). The detector is a 12-bit digital CCD with $1024 \times 1360$ pixels with $4.65 \mu \mathrm{m}$ pitches (PCO Pixel Fly). In the set-up, the laser is a $2 \omega N d Y A G$ and the reference wave is smooth and plane and the curvature radius of the numerical reconstructing wave is adjusted according to the wanted transversal magnification. 


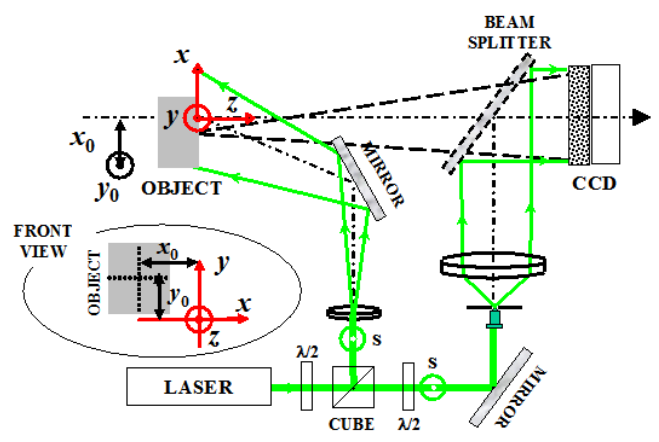

Fig. 1. Experimental setup

\section{Experimental results}

As an illustration, the object under consideration is the bronze medal of the running race " $20 \mathrm{~km}$ de Paris 2000" which is $60 \mathrm{~mm}$ in diameter. The distance for the recording is set to $d_{0}=1500 \mathrm{~mm}$ and the object is translated perpendicularly to the optical axis of quantities $\left(x_{0}=53.6 \mathrm{~mm}, y_{0}=-51.9 \mathrm{~mm}\right)$ thus producing off axis spatial frequencies $\left(u_{0}, v_{0}\right)=\left(x_{0} / \lambda d_{0}, y_{0} / \lambda d_{0}\right) \approx\left(67.2 \mathrm{~mm}^{-1},-65.1 \mathrm{~mm}^{-1}\right)$. Fig. 3 shows the reconstructed object obtained with algorithm of Eq. 1 with $K=L=2048, \gamma=0.158$ (left) and $\gamma=0.14$ (right). For $\gamma=0.158$, parameters are $R_{c}=-281.4 \mathrm{~mm}, d_{R}=-237 \mathrm{~mm}$ and for $\gamma=0.14$ they are $R_{c}=-244.2 \mathrm{~mm}, d_{R}=-210 \mathrm{~mm}$.
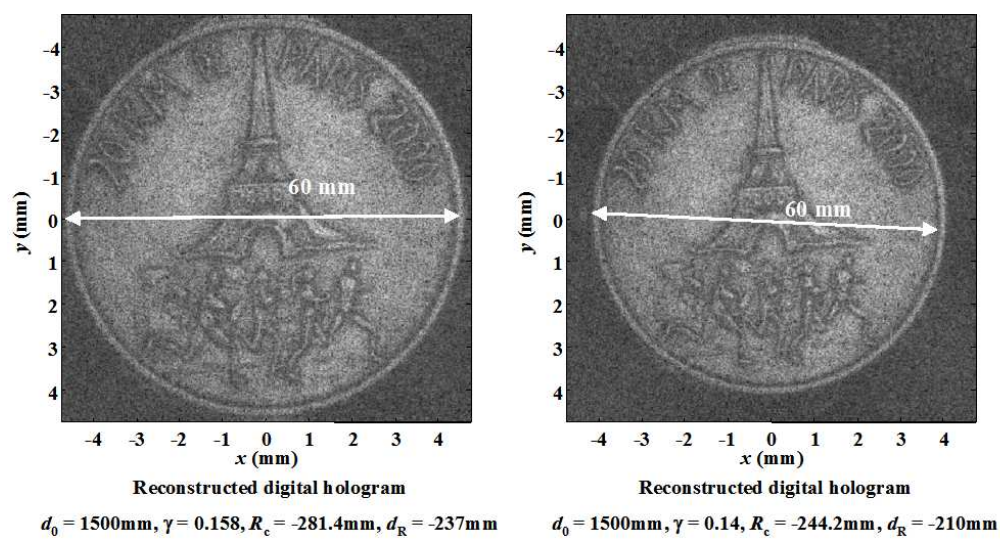

Fig. 2. Reconstruction of a monochrome digital hologram of a medal $60 \mathrm{~mm}$ in diameter $(\lambda=532 \mathrm{~nm})$

\section{Extension to digital color holography}

The proposed algorithms are also suitable for digital color holography. Experimental results are provided in the case of digital two-color holograms. The set-up is modified to include a second Mach-Zehnder interferometer using a HeNe laser (Fig. 3). Each laser beam illuminates the object under interest with illuminating angles $\theta_{R}$ and $\theta_{G}$ respectively for the red and green lines (in the setup $\theta_{R}=-\theta_{G}=\theta$ ). The smooth and plane reference waves are produced through the two spatial filters (SF1 and SF2). Since the monochrome sensor is not able to simultaneously record the two colors at each pixel, the spatial frequencies of the reference waves $(R$ and $G)$ are adjusted so that the twocolor holograms are spatially multiplexed in the field of view. Thus, the off-line holographic 
recording is carried out using the two spatial filters in which each collimating lens is displaced out of the afocal axis by means of two micrometric transducers (not shown in Fig. 4).

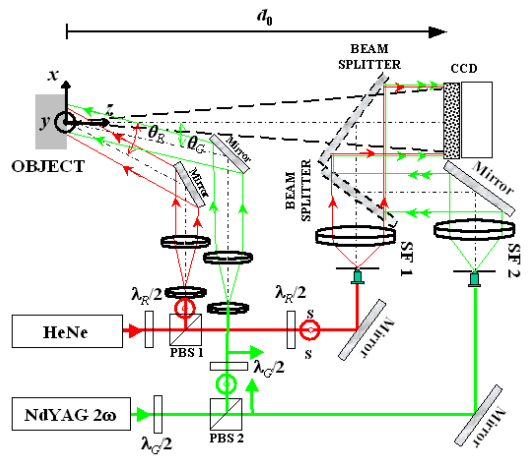

Fig. 3. Set-up for digital two-color holography

Using Eq. 2, Fig. 4 shows two-color holographic reconstructions of objects with various shapes, simultaneously illuminated with the HeNe laser and the $2 \omega$ NdYAD laser. Fig. 4 also shows CCD images of the two objects illuminated in the same conditions with the two lasers. It is satisfactorily faithful pictures compared to the ordinary pictures, taken by the CCD with an imaging lens.

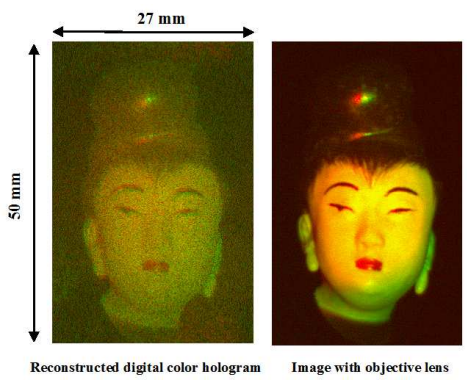

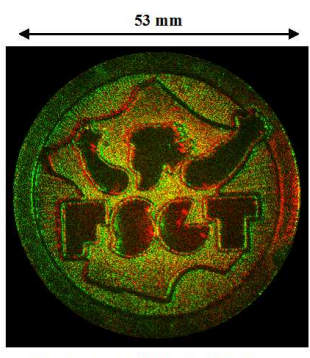

Reconstructed digital color hologram

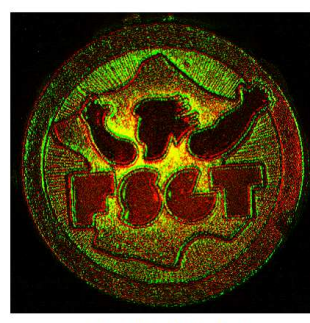

Image with objective lens

Fig. 4. Example for 2-color digital holograms of large objects

\section{Multidimensional deformation measurement with two-color digital holography}

Digital holographic interferometry is the tool that can provide non contact and global measurements with a sub-micrometer range sensibility and it is highly suitable for the investigation of mechanical properties of some mechanical component or assembly. In order to do this, the phase of two reconstructed wave fields recorded at different loadings of the object are computed from the complex amplitude and the displacement field is obtained from the difference of the two optical phases. Particularly, applications in the field of mechanics require a multiple component measurement of the displacement field of the studied structure. In the past, Pedrini proposed a twodimensional digital double pulse holographic system with a twin sensitivity corresponding to two illumination directions [6]. The deformation along the two sensitivity vectors was recorded simultaneously using two CCD detectors. Lastly, the concept of spatial multiplexing was adapted to speckle interferometry with a spatial carrier [7-8], for a three-dimensional dynamic deformation evaluation. In these papers, the incoherent addition of the multiplexed holograms is performed by use of a delay line between each Mach-Zehnder interferometer; indeed, some depolarisation occurs due to the nature of the material composing the object. Note that pure polarizing interferometers can be used in other cases [9]. However, such strategies require a highly complicated set-up and a 
powerful laser source. The method proposed in this paper is based on the two-color-spatialmultiplexing of digital holograms and allows a simultaneous in-plane and out-of-plane deformation measurement. The benefits of such approach are that the is no need for high power laser $(100 \mathrm{~mW}$ DPSS lasers or HeNe lasers can be used) and there is no interference crossing between colors. Algorithms proposed in the previous section can be applied to two-color holograms. The case exposed here is that of an aluminium wafer $40 \mathrm{~mm}$ in diameter. Fig. 5 shows the two color reconstructed object. Fig. 5a shows the full field hologram reconstructed using the classical Fresnel transform; it can be seen clearly that the size of the two multiplexed holograms varies with the wavelength (on the right is localized the green object $(G)$ and on the left the red one $(R)$ ). Fig. $5 \mathrm{~b}$ shows the $G$ object reconstructed with one of the proposed algorithm (Eq. 2) and Fig. 5c shows the $R$ object obtained with the same algorithm. Note that the object sizes are the same and that the speckle in the image is bigger in the red channel than in the green channel, as can be expected.
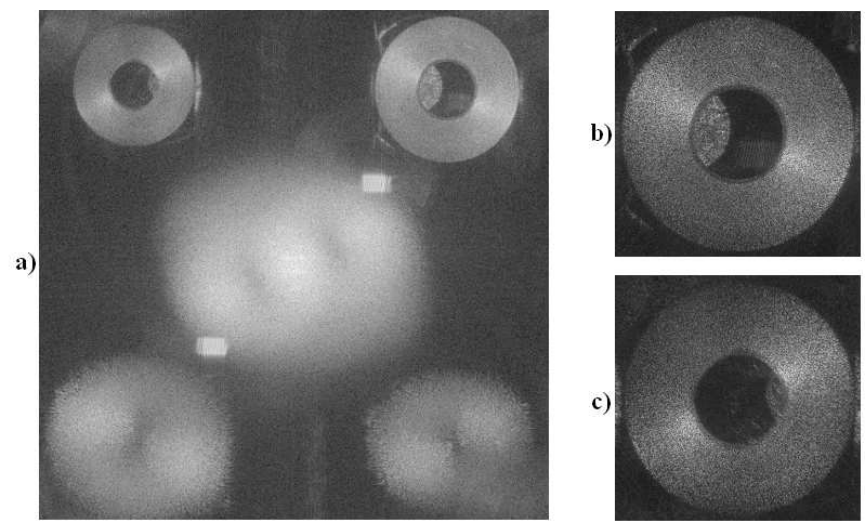

Fig. 5. Reconstructed object; a) full field hologram, b) green object c) red object (reconstructed horizon : $2048 \times 2048$ pixels)

When applying a loading along the $x$ direction, the object is deformed. The optical phase change before and after the loading can be computed from the numerical reconstruction of the object. Fig. 6 shows such phase change obtained at two different mechanical loading of the object. Basically, the phase change is obtained modulo $2 \pi$ and exhibits phase jumps that must be removed. Fig. 6 , left, shows the phase change obtained when computing the object using a wavelength-dependant-zeropadding Fresnel transform with $1024 \times 1210$ pixels [5]. The useful part of the field includes $300 \times 300$ pixels. It can be seen some noise in the phase map. Fig. 6, right, shows the phase change obtained when computing the object using algorithm of Eq. 2. The useful part of the field includes now $2048 \times 2048$ pixels. The significant result is that the noise has been decreased between the two results. That is certainly due to the fact that the algorithm acts as a zero-padding algorithm using a very high number of data points. Thus, the "definition" of the image is quite better than with Fresnel transform, thus increasing the signal-to-noise ratio. So, as a conclusion, the proposed method is of general interest since it also increases the signal to noise ratio in phase measurements giving a more contrasted phase map. 

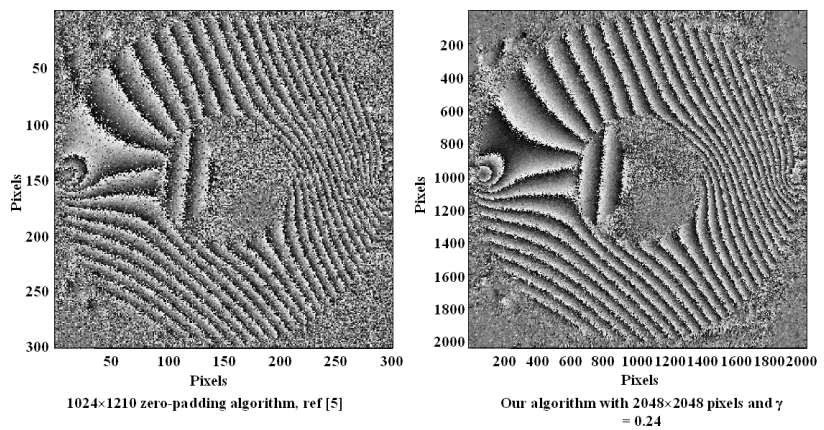

Fig. 6. Comparison between zero-padding algorithm [5] and algorithms of Eq. 2

The two-color set-up is devoted to the case of simultaneous two-dimensional deformation measurements. With the recording of color holograms before and after the loading, the phase map of the deformation can be computed along the red channel, i.e. $\Delta \varphi_{R}$, and along the green channel, i.e. $\Delta \varphi_{G}$. Then phase changes are unwrapped, i.e. the $2 \pi$ phase jumps seen in Fig. 6 are removed. After that, the displacement field along the $x$ and $z$ directions can be computed using the $R$ and $G$ phase changes according to these two equations:

$$
u_{x}=\frac{1}{2 \pi \sin \theta}\left(\lambda_{R} \Delta \varphi_{R}-\lambda_{G} \Delta \varphi_{G}\right)
$$

and

$$
u_{z}=-\frac{1}{2 \pi(1+\cos \theta)}\left(\lambda_{R} \Delta \varphi_{R}+\lambda_{G} \Delta \varphi_{G}\right)
$$

In the set-up the illuminating angle is adjusted to be $\theta=45^{\circ}$. We applied algorithm of Eq. 2 to the case of the mechanical loading of the aluminium wafer. Recording of a two-color hologram was performed before any loading; then, loading of the object along the $x$ direction (horizontal direction in Fig. 6) was applied. After extracting the red and green phases with the set of color spatially multiplexed holograms, the in plane and out of plane displacements are computed according to previous equations. Fig. 7 and 8 show the in-plane and the out-of-plane displacement fields obtained after loading of the object along $x$.

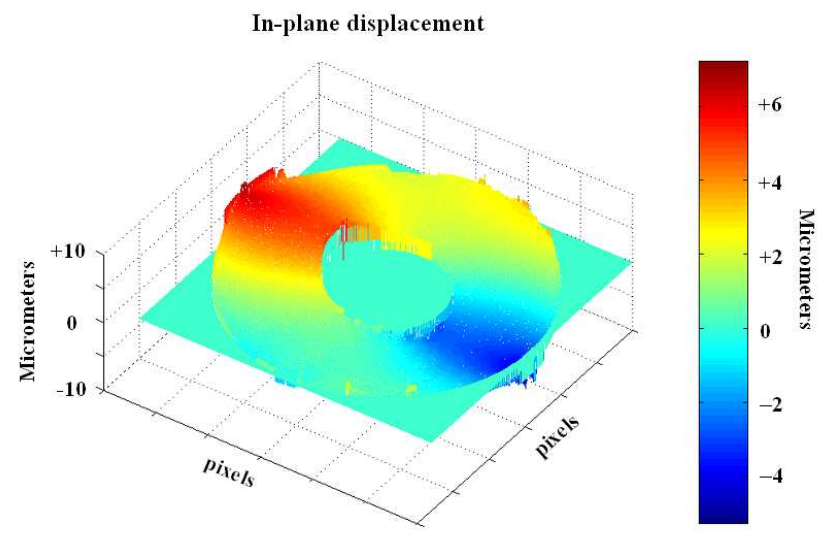

Fig. 7. In-plane displacement field computed from two-color digital holograms 


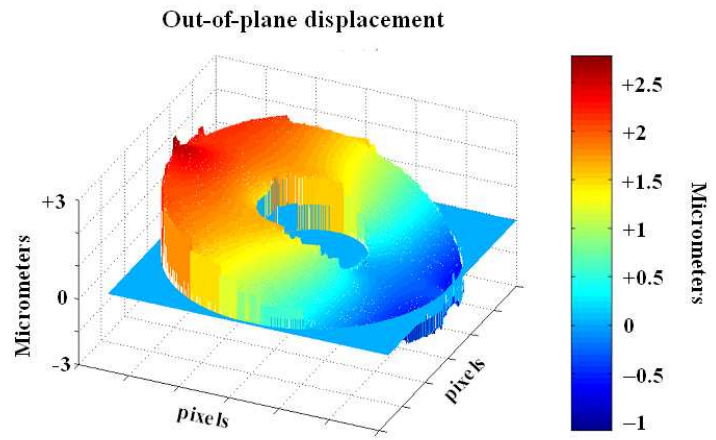

Fig. 8. Out-of-plane displacement field computed from two-color digital holograms

\section{Conclusion}

The paper has presented two numerical methods for reconstructing large objects using a convolution method with an adjustable magnification based on the use of a virtual spherical reconstructing wave. The filtering is realized using a modified version of the angular spectrum transfer function or using the spatially phase biased Fresnel response of free space propagation. Experimental results presented in the case of monochrome and two-color recordings confirm the suitability of the proposed methods. Application of the method in the case of simultaneous two-dimensional deformation measurement of an object submitted to a mechanical loading is proposed and it is shown that the signal-to-noise ratio in phase changes is increased.

\section{References}

1. Th. Kreis, M. Adams, W. Jüptner, Proc. SPIE 3098, 224 (1997).

2. D. Mas, J. Perez, C. Hernandez, C. Vazquez, J.J. Miret, C. Illueca, Opt. Comm. 227, 245 (2003).

3. J.C. Li, Z. Peng, Y. Fu, Opt. Comm. 280, 243 (2007).

4. L. Yu, M.K. Kim, Opt. Lett. 30, 2092 (2005).

5. P. Ferraro, S. De Nicola, G. Coppola, A. Finizio, D. Alfieri, G. Pierattini, Opt. Lett. 29, 854 (2004).

6. G. Pedrini, H.J. Tiziani, Measurement 18, 251-260 (1995).

7. G. Pedrini, Y.L. Zou, H.J. Tiziani, Appl. Opt. 36, 786-792 (1997).

8. S. Schedin, G. Pedrini, H.J. Tiziani, F.M. Santoyo, Appl. Opt. 38, 7056-7062 (1999).

9. P. Picart, B. Diouf, E. Lolive, J.-M. Berthelot, Opt. Eng. 43, 1169-1176 (2004). 